\title{
COMBINED DEM EXTRACTION METHOD FROM STEREOSAR AND INSAR
}

\author{
Z. Zhao ${ }^{\text {a, } *, ~ J . ~ X . ~ Z h a n g ~}{ }^{\text {a }}$, M. Y. Duan ${ }^{\text {a }}$, G. M. Huang ${ }^{\text {a }}$, S. C. Yang ${ }^{\text {a }}$ \\ ${ }^{\text {a }}$ Chinese Academy of Surveying and Mapping, No.28 Lianhuachi West Road, Beijing 100830, China - zhengzhaochina@163.com
}

Commission VI, WG VI/4

KEY WORDS: DEM, StereoSAR, InSAR, Adjustment, Model, Extraction

\begin{abstract}
:
A pair of SAR images acquired from different positions can be used to generate digital elevation model (DEM). Two techniques exploiting this characteristic have been introduced: stereo SAR and interferometric SAR. They permit to recover the third dimension (topography) and, at the same time, to identify the absolute position (geolocation) of pixels included in the imaged area, thus allowing the generation of DEMs. In this paper, StereoSAR and InSAR combined adjustment model are constructed, and unify DEM extraction from InSAR and StereoSAR into the same coordinate system, and then improve three dimensional positioning accuracy of the target. We assume that there are four images 1, 2, 3 and 4. One pair of SAR images 1,2 meet the required conditions for InSAR technology, while the other pair of SAR images 3,4 can form stereo image pairs. The phase model is based on InSAR rigorous imaging geometric model. The master image 1 and the slave image 2 will be used in InSAR processing, but the slave image 2 is only used in the course of establishment, and the pixels of the slave image 2 are relevant to the corresponding pixels of the master image 1 through image coregistration coefficient, and it calculates the corresponding phase. It doesn't require the slave image in the construction of the phase model. In Range-Doppler (RD) model, the range equation and Doppler equation are a function of target geolocation, while in the phase equation, the phase is also a function of target geolocation. We exploit combined adjustment model to deviation of target geolocation, thus the problem of target solution is changed to solve three unkonwns through seven equations. The model was tested for DEM extraction under spaceborne InSAR and StereoSAR data and compared with InSAR and StereoSAR methods respectively. The results showed that the model delivered a better performance on experimental imagery and can be used for DEM extraction applications.
\end{abstract}

\section{INTRODUCTION}

Synthetic Aperture Radar Interferometry (InSAR) is a landmark achievement with the development of SAR, and has a great potential in development and application. As fastest growing technologies in Earth science and remote sensing, InSAR is a hot research topic as well. With the fast development in SAR sensors, it provides possible massive, rapid and high-resolution SAR acquisitions for InSAR applications such as topographic mapping, surface deformation, hazard monitoring, glaciers movement, forest resources investigation, agriculture monitoring and wetland change mapping etc ( $\mathrm{Lu}, \mathrm{Z}$. et al., 2014). Nevertheless, how to effectively generate a high accuracy digital elevation model (DEM)in sophisticated terrain area using InSAR is still a hot research interest.

In sophisticated terrain regions, there are many problems in DEM extraction using InSAR (Zhang, J.X. et al., 2012). Firstly, due to the SAR side-looking imaging model, a large shadow and serious layover area can be expected in the steep terrain area, which cause real terrain information loss and holes in DEM results. Secondly, in fractured terrain regions, the low coherence in interferograms and accuracy in DEM extraction are unable to support the practical demands. The above problems are even worse for DEM extraction using InSAR (Crosetto M. et al., 2000; Deng S et al., 2011; Eldhuset K et al., 2013). Regarding to the above problems, research strategies and solutions are proposed in this paper. StereoSAR assisted InSAR, in sophisticated terrain area, this method exploits the advantages of InSAR and StereoSAR respectively to extract DEM comprehensively. Furthermore, StereoSAR and InSAR combined adjustment model are constructed and proposed as well. This model can unify DEM extraction from InSAR and StereoSAR into the same coordinate system, and then improve three dimensional positioning accuracy of the target.

\section{STEREOSAR AND INSAR COMBINED ADJUSTMENT MODEL}

\subsection{Overview}

In the traditional SAR system, the target position along range direction is determined by the amount of SAR pulse propagation time between the target and the return radar, and the target position along azimuth direction is determined by the Doppler shift. In terms of geometry, location of the target is the intersection of the cone which takes the antenna as the center, the radar distance as the radius of the sphere and along the velocity vector as the axis, and angle is proportional to the Doppler frequency. The target point on the SAR image is located on a circle that passes through the target and is perpendicular to the plane of the sensor flight direction.

In InSAR processing, the interferometric phase is provided by using two or more SAR images, and it provides the technical means for obtaining the 3D position information of the target. Measured spatial target is not only the intersection of range sphere and Doppler cone, is but also needed to meet the

* Corresponding author 
interferometric phase equation (Bamler R. et al., 1998) Therefore, the spatial measured target point is the intersection of range sphere, Doppler cone and phase cone. Among them, the axis of Doppler cone denotes velocity vector, and the axis of phase cone denotes baseline vector. It's shown in figure 1 . To be sure, although in the actual situation, the solution of interferometric phase equation is a hyperbolic surface, but in general application, the spherical wave of electromagnetic wave is considered to be a plane wave, and the hyperbolic plane is simplified to a conical surface.

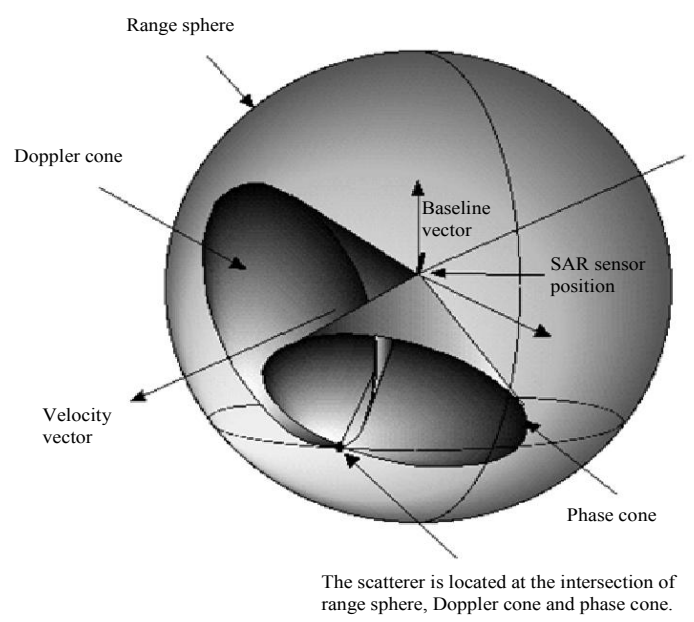

Figure 1. InSAR processing geometry

\subsection{StereoSAR and InSAR combined adjustment model}

Before DEM fusion generated from stereogrammetry and InSAR, in order to take stereo DEM and InSAR DEM into the same coordinate system, StereoSAR and InSAR combined adjustment model is constructed. StereoSAR and InSAR Range Doppler equations and phase equations are combined to uniform adjustment.

We assume that there are four images 1,2, 3 and 4. One pair of SAR images 1,2 meet the required conditions for InSAR technology, and image 1 is the master image, then image 2 is the slave image, while the other pair of SAR images 3,4 can form stereo image pairs, and image 3 is the left image, then image 4 is the right image. The phase model is based on InSAR rigorous imaging geometric model. The master image 1 and the slave image 2 will be used in InSAR processing, but the slave image 2 is only used in the course of establishment, and the pixels of the slave image 2 are relevant to the corresponding pixels of the master image 1 through image coregistration coefficient, and it calculates the corresponding phase. It doesn't require the slave image in the construction of the phase model. In Range-Doppler (RD) model (Curlander, 1982), the range equation and Doppler equation are a function of target geolocation $\left(X_{t}, Y_{t}, Z_{t}\right)$, while in the phase equation, the phase is also a function of target geolocation $\left(X_{t}, Y_{t}, Z_{t}\right)$. We exploit combined adjustment model to deviation of target geolocation $\left(X_{t}, Y_{t}, Z_{t}\right)$, thus the problem of target solution is changed to solve three unkonwns through seven equations. Then the combined adjustment model is as follows:

$$
\begin{gathered}
F_{1}=\varphi+\frac{4 \pi}{\lambda}\left(R_{1}-R_{2}\right) \\
F_{2}=R_{1}^{\prime}-R_{1} \\
F_{3}=-\frac{2}{\lambda} \frac{\left(X_{S_{1}}-X_{t}\right) \cdot X_{V_{1}}+\left(Y_{S_{1}}-Y_{t}\right) \cdot Y_{V_{1}}+\left(Z_{S_{1}}-Z_{t}\right) \cdot Z_{V_{1}}}{R_{1}^{\prime}} \\
F_{4}=R_{3}^{\prime}-R_{3} \\
F_{5}=-\frac{2}{\lambda} \frac{\left(X_{S_{3}}-X_{t}\right) \cdot X_{V_{3}}+\left(Y_{S_{3}}-Y_{t}\right) \cdot Y_{V_{3}}+\left(Z_{S_{3}}-Z_{t}\right) \cdot Z_{V_{3}}}{R_{3}^{\prime}} \\
F_{6}=R_{4}^{\prime}-R_{4} \\
F_{7}=-\frac{2}{\lambda} \frac{\left(X_{S_{4}}-X_{t}\right) \cdot X_{V_{4}}+\left(Y_{S_{4}}-Y_{t}\right) \cdot Y_{V_{4}}+\left(Z_{S_{4}}-Z_{t}\right) \cdot Z_{V_{4}}}{R_{4}^{\prime}}
\end{gathered}
$$

where

$$
\begin{aligned}
& R_{1}^{\prime}=\sqrt{\left(X_{s_{1}}-X_{t}\right)^{2}+\left(Y_{s_{1}}-Y_{t}\right)^{2}+\left(Z_{s_{1}}-Z_{t}\right)^{2}} \\
& R_{2}=\sqrt{R_{1}^{2}+B^{2}-2 R_{1} B \sin (\theta-\alpha)}
\end{aligned}
$$

Due to

$$
\frac{\partial F_{1}}{\partial X_{t}}=\frac{4 \pi}{\lambda}\left(\frac{\partial R_{1}}{\partial X_{t}}-\frac{\partial R_{2}}{\partial X_{t}}\right)
$$

where

$$
\begin{aligned}
\frac{\partial R_{1}}{\partial X_{t}}= & \frac{X_{t}-X_{S_{1}}}{R_{1}^{\prime}} \\
\frac{\partial R_{2}}{\partial X_{t}}= & \frac{1}{2 R_{2}}\left(2 R_{1} \cdot \frac{\partial R_{1}}{\partial X_{t}}-2 B \cdot \sin (\theta-\alpha) \cdot \frac{\partial R_{1}}{\partial X_{t}}\right) \\
& =\frac{\left[R_{1}-B \cdot \sin (\theta-\alpha)\right] \cdot \frac{X_{t}-X_{S_{1}}}{R_{1}^{\prime}}}{R_{2}} \\
& =\left[R_{1}-B \cdot \sin (\theta-\alpha)\right] \cdot \frac{X_{t}-X_{S_{1}}}{R_{1}^{\prime} \cdot R_{2}}
\end{aligned}
$$

It can be deduced.

$$
\frac{\partial F_{1}}{\partial X_{t}}=\frac{4 \pi}{\lambda} \cdot \frac{X_{t}-X_{S_{1}}}{R_{1}^{\prime}}\left[1-\frac{R_{1}-B \cdot \sin (\theta-\alpha)}{R_{2}}\right]
$$

Similarly,

$$
\begin{aligned}
& \frac{\partial F_{1}}{\partial Y_{t}}=\frac{4 \pi}{\lambda} \cdot \frac{Y_{t}-Y_{S_{1}}}{R_{1}^{\prime}}\left[1-\frac{R_{1}-B \cdot \sin (\theta-\alpha)}{R_{2}}\right] \\
& \frac{\partial F_{1}}{\partial Z_{t}}=\frac{4 \pi}{\lambda} \cdot \frac{Z_{t}-Z_{S_{1}}}{R_{1}^{\prime}}\left[1-\frac{R_{1}-B \cdot \sin (\theta-\alpha)}{R_{2}}\right] \\
& \frac{\partial F_{2}}{\partial X_{t}}=\frac{\partial R_{1}^{\prime}}{\partial X_{t}}=\frac{X_{t}-X_{S_{1}}}{R_{1}^{\prime}} \\
& \frac{\partial F_{2}}{\partial Y_{t}}=\frac{\partial R_{1}^{\prime}}{\partial Y_{t}}=\frac{Y_{t}-Y_{S_{1}}}{R_{1}^{\prime}} \\
& \frac{\partial F_{2}}{\partial Z_{t}}=\frac{\partial R_{1}^{\prime}}{\partial Z_{t}}=\frac{Z_{t}-Z_{S_{1}}}{R_{1}^{\prime}}
\end{aligned}
$$




$$
\begin{aligned}
& \frac{\partial F_{3}}{\partial X_{t}}=\frac{2}{\lambda}\left[\frac{X_{V_{1}}}{R_{1}^{\prime}}-\frac{\left(X_{S_{1}}-X_{t}\right)^{2} \cdot X_{V_{1}}}{R_{1}^{\prime 3}}\right] \\
& \frac{\partial F_{3}}{\partial Y_{t}}=\frac{2}{\lambda}\left[\frac{Y_{V_{1}}}{R_{1}^{\prime}}-\frac{\left(Y_{S_{1}}-Y_{t}\right)^{2} \cdot Y_{V_{1}}}{R_{1}^{\prime 3}}\right] \\
& \frac{\partial F_{3}}{\partial Z_{t}}=\frac{2}{\lambda}\left[\frac{Z_{V_{1}}}{R_{1}^{\prime}}-\frac{\left(Z_{S_{1}}-Z_{t}\right)^{2} \cdot Z_{V_{1}}}{R_{1}^{\prime 3}}\right] \\
& \frac{\partial F_{4}}{\partial X_{t}}=\frac{\partial R_{3}^{\prime}}{\partial X_{t}}=\frac{X_{t}-X_{S_{3}}}{R_{3}^{\prime}} \\
& \frac{\partial F_{4}}{\partial Y_{t}}=\frac{\partial R_{3}^{\prime}}{\partial Y_{t}}=\frac{Y_{t}-Y_{S_{3}}}{R_{3}^{\prime}} \\
& \frac{\partial F_{4}}{\partial Z_{t}}=\frac{\partial R_{3}^{\prime}}{\partial Z_{t}}=\frac{Z_{t}-Z_{S_{3}}}{R_{3}^{\prime}} \\
& \frac{\partial F_{5}}{\partial X_{t}}=\frac{2}{\lambda}\left[\frac{X_{V_{3}}}{R_{3}^{\prime}}-\frac{\left(X_{S_{3}}-X_{t}\right)^{2} \cdot X_{V_{3}}}{R_{3}^{\prime 3}}\right] \\
& \frac{\partial F_{5}}{\partial Y_{t}}=\frac{2}{\lambda}\left[\frac{Y_{V_{3}}}{R_{3}^{\prime}}-\frac{\left(Y_{S_{3}}-Y_{t}\right)^{2} \cdot Y_{V_{3}}}{R_{3}^{\prime 3}}\right] \\
& \frac{\partial F_{5}}{\partial Z_{t}}=\frac{2}{\lambda}\left[\frac{Z_{V_{3}}}{R_{3}^{\prime}}-\frac{\left(Z_{S_{3}}-Z_{t}\right)^{2} \cdot Z_{V_{3}}}{R_{3}^{\prime 3}}\right] \\
& \frac{\partial F_{6}}{\partial X_{t}}=\frac{\partial R_{4}^{\prime}}{\partial X_{t}}=\frac{X_{t}-X_{S_{4}}}{R_{4}^{\prime}} \\
& \frac{\partial F_{6}}{\partial Y_{t}}=\frac{\partial R_{4}^{\prime}}{\partial Y_{t}}=\frac{Y_{t}-Y_{S_{4}}}{R_{4}^{\prime}} \\
& \frac{\partial F_{6}}{\partial Z_{t}}=\frac{\partial R_{4}^{\prime}}{\partial Z_{t}}=\frac{Z_{t}-Z_{S_{4}}}{R_{4}^{\prime}} \\
& \frac{\partial F_{7}}{\partial X_{t}}=\frac{2}{\lambda}\left[\frac{X_{V_{4}}}{R_{4}^{\prime}}-\frac{\left(X_{S_{4}}-X_{t}\right)^{2} \cdot X_{V_{4}}}{R_{4}^{\prime 3}}\right] \\
& \frac{\partial F_{7}}{\partial Y_{t}}=\frac{2}{\lambda}\left[\frac{Y_{V_{4}}}{R_{4}^{\prime}}-\frac{\left(Y_{S_{4}}-Y_{t}\right)^{2} \cdot Y_{V_{4}}}{R_{4}^{\prime 3}}\right] \\
& \frac{\partial F_{7}}{\partial Z_{t}}=\frac{2}{\lambda}\left[\frac{Z_{V_{4}}}{R_{4}^{\prime}}-\frac{\left(Z_{S_{4}}-Z_{t}\right)^{2} \cdot Z_{V_{4}}}{R_{4}^{\prime 3}}\right]
\end{aligned}
$$

Because of the nonlinear equation, the equation must be linearized, and the initial value is given, and then it carries on iterative solution.

The error equations of single point are as follows:

$$
V=B X-L
$$

$$
\begin{aligned}
& \text { where } \quad V=\left(\begin{array}{c}
V_{1} \\
V_{2} \\
V_{3} \\
V_{4} \\
V_{5} \\
V_{6} \\
V_{7}
\end{array}\right) \\
& B=\left(\begin{array}{ccc}
\frac{\partial F_{1}}{\partial X_{t}} & \frac{\partial F_{1}}{\partial Y_{t}} & \frac{\partial F_{1}}{\partial Z_{t}} \\
\vdots & \ddots & \vdots \\
\frac{\partial F_{7}}{\partial X_{t}} & \frac{\partial F_{7}}{\partial Y_{t}} & \frac{\partial F_{7}}{\partial Z_{t}}
\end{array}\right) \\
& X=\left(\begin{array}{l}
\Delta X_{t} \\
\Delta Y_{t} \\
\Delta Z_{t}
\end{array}\right) \\
& L=\left(\begin{array}{l}
-F_{1(0)} \\
-F_{2(0)} \\
-F_{3(0)} \\
-F_{4(0)} \\
-F_{5(0)} \\
-F_{6(0)} \\
-F_{7(0)}
\end{array}\right) \\
& F_{1(0)}=\varphi_{0}+\frac{4 \pi}{\lambda}\left(R_{1(0)}-R_{2(0)}\right) \\
& F_{2(0)}=R_{1(0)}^{\prime}-R_{1(0)} \\
& F_{3(0)}=-\frac{2}{\lambda} \frac{\left(X_{S_{1}}-X_{t(0)}\right) \cdot X_{V_{1}}+\left(Y_{S_{1}}-Y_{t(0)}\right) \cdot Y_{V_{1}}+\left(Z_{S_{1}}-Z_{t(0)}\right) \cdot Z_{V_{1}}}{R_{1(0)}^{\prime}} \\
& F_{4(0)}=R_{3(0)}^{\prime}-R_{3(0)} \\
& F_{5(0)}=-\frac{2}{\lambda} \frac{\left(X_{S_{3}}-X_{t(0)}\right) \cdot X_{V_{3}}+\left(Y_{S_{3}}-Y_{t(0)}\right) \cdot Y_{V_{3}}+\left(Z_{S_{3}}-Z_{t(0)}\right) \cdot Z_{V_{3}}}{R_{3(0)}^{\prime}} \\
& F_{6(0)}=R_{4(0)}^{\prime}-R_{4(0)} \\
& F_{7(0)}=-\frac{2}{\lambda} \frac{\left(X_{S_{4}}-X_{t(0)}\right) \cdot X_{V_{4}}+\left(Y_{S_{4}}-Y_{t(0)}\right) \cdot Y_{V_{4}}+\left(Z_{S_{4}}-Z_{t(0)}\right) \cdot Z_{V_{4}}}{R_{4}^{\prime}}
\end{aligned}
$$

\section{TEST AND RESULTS}

DEMs are generated from InSAR techniques, stereogrammetry techniques and combined techniques in Deqin, Yunnan Province. InSAR related results are shown in figure 2 including DEM, layover and shadow mask and coherence map. DEM extracted through ascending stereogrammetry and descending stereogrammetry are shown in figure 3 . Combined adjustment results are shown in figure 4. 

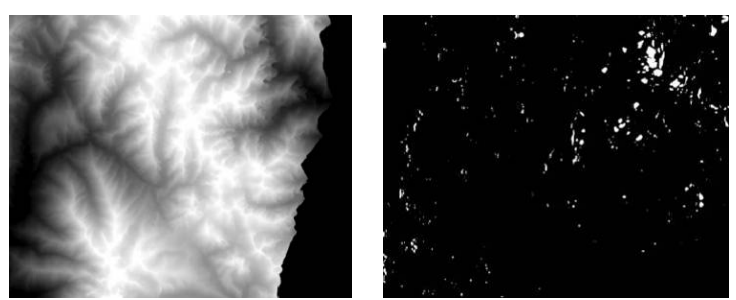

(a) DEM extracted through InSAR(b) layover and shadow mask

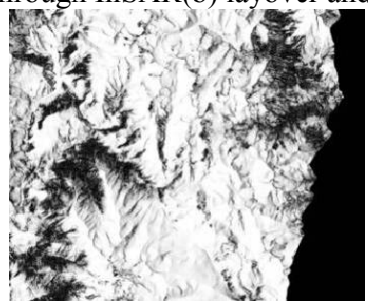

(c) coherence map

Figure 2. InSAR related results

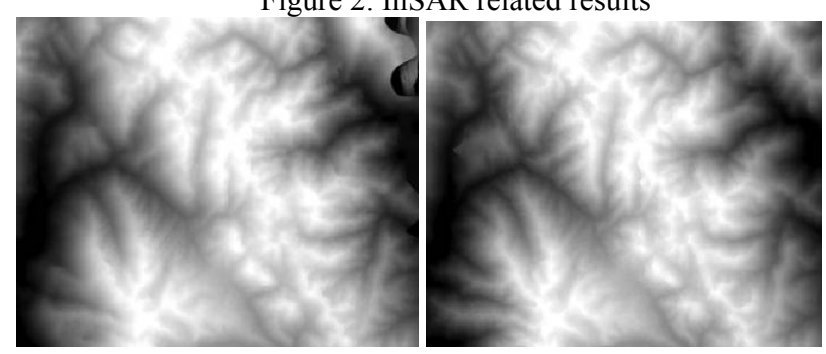

(a)DEM extracted through ascending StereoSAR (b)descending StereoSAR

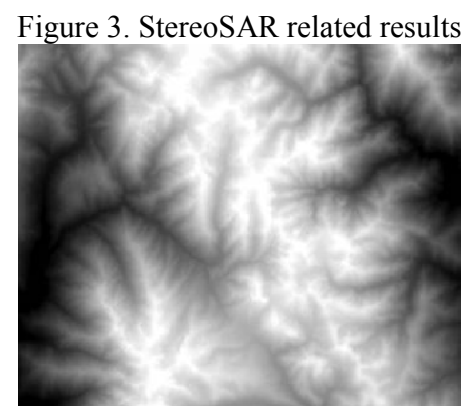

Figure 4. Combined adjustment results

The DEM accuracy was checked by 42 GPS field survey points, and the error of combined DEM is about \pm 9.1 meters. The DEM accuracy comparison is shown in Table 1.

\begin{tabular}{|c|c|c|c|}
\hline Items & $\begin{array}{c}\text { StereoDEM } \\
(\mathrm{m})\end{array}$ & $\begin{array}{c}\text { InSAR DEM } \\
(\mathrm{m})\end{array}$ & Combined(m) \\
\hline Maximum error & 27.3 & 37.4 & 14.9 \\
\hline Minimum error & 3.0 & 0.6 & 0.6 \\
\hline RMSE & \pm 16.69 & \pm 16.67 & \pm 9.1 \\
\hline
\end{tabular}

Table 1. DEM accuracy comparison

\section{CONCLUSIONS}

The combined method can not only complement each other to generate DEM from StereoSAR and InSAR respectively, but also improve the accuracy of DEM.

In the complex terrain, the InSAR and StereoSAR are fully played their respective advantages. StereoSAR and InSAR combined adjustment model are constructed, StereoSAR and InSAR Range Doppler equations and phase equations are combined to uniform adjustment, and unify DEM extraction from InSAR and StereoSAR into the same coordinate system, and then improve three dimensional positioning accuracy of the target. Finally, the combined InSAR DEM and StereoSAR DEM can effectively compensate for the effect of InSAR on the loss of coherence and the effect of StereoSAR on the layover and the shadow.

\section{ACKNOWLEDGEMENTS}

This research was Funded by the 2015 annual remote sensing young talents of innovation fundation; National basic surveying and mapping project: Airborne SAR large scale mapping (A1505); Surveying\&Mapping and Geoinformation Research in the Public Interest(201412002).

\section{REFERENCES}

Bamler R, Hartl P. 1998. Synthetic aperture radar interferometry. Inverse Problems, 14(4): R1-R54.

Crosetto M, Pérez Aragues F. 2000. Radargrammetry and SAR Interferometry for DEM Generation Validation and Data Fusion. EUROPEAN SPACE AGENCY-PUBLICATIONS-ESA SP, 450: 367-372.

Curlander J C. 1982. Location of spaceborne SAR imagery. Geoscience and Remote Sensing, IEEE Transactions on, 1982 (3): 359-364.

Deng S, Zhang J, Li P, et al. 2011. DEM Fusion and Its Application in Mapping Topography of Complex Areas. In: Image and Data Fusion (ISIDF), 2011 International Symposium on. IEEE, 2011: 1-4.

Eldhuset K, Weydahl D J. 2013. Using stereo SAR and InSAR by combining the COSMO-SkyMed and the TanDEM-X mission satellites for estimation of absolute height. International Journal of Remote Sensing, 4(23): 8463-8474.

Lu, Z., and Zhang, L., 2014. Frontiers of Radar Remote Sensing. Photogrammetric Engineering \& Remote Sensing, 80 (1), 5-13.

Zhang, J.X., Zhao, Z., Huang, G.M., and Lu, Z., 2012. CASMSAR: An Integrated Airborne SAR Mapping System. Photogrammetric Engineering \& Remote Sensing, 78 (11), 1110-1114. 